\title{
Emergency Department Overuse: A Case Report
}

\author{
Ensar Durmuş* \\ Department of Medicine, Sakarya Ministry of Health Sakarya University, Turkey
}

\begin{abstract}
Objective: The present study aims to investigate a patient with a large number of admissions to adult emergency service, to understand the cost of the patient to the public through the number of admissions and the invoice amount, and to reveal the solutions available for such patients.

Introduction: Emergency services are the units providing free health services with intensive patient admissions in Turkey. Emergency department admissions were gradually increasing in recent years, with claims that emergency services are being abused by patients.

Results: Our patient was found to admit to the emergency department 266 times in two years for reasons such as substance abuse, excessive drug use, and self-harm, and he was hospitalized 4 times in the service and 9 times in the intensive care unit. The invoice total of the examination and treatment bill was 21,763.05 Turkish Lira.

Conclusion: Patients with addictions and psychiatric disorders have the potential to harm themselves and cause multiple emergency service admissions, leading to increased workload in the emergency service. It may be necessary to address these patients differently than other patients and to conduct a more detailed examination.
\end{abstract}

Keywords: Emergency service • Abuse • Substance abuse

\section{Introduction}

Emergency services in Turkey are the units with intensive patient admissions, working 24/7 and providing free health services, regardless of whether the patients have any health insurance. Admissions for emergency services are increasing in Turkey, as in other countries with each passing year. The number of emergency admissions in the UK increased from 15.4 million to 18.4 million in 4 years, from 2007-2008 to 2011-2012 [1]. In Turkey, however, the total number of emergency examinations performed in public hospitals in 2017 was 101,473,472. Of this number, 91,991,243 were the adult emergency and $9,482,499$ were the pediatric emergency examinations. Of the total number of all examinations conducted in $2017,28.36 \%$ was emergency examinations [2]. This increases unnecessary health expenses as well as the workload. Overuse of emergency services in the US causes a waste of $\$ 38$ billion a year [3]. In addition, the number of emergency admissions is increasing day by day and emergency service admissions have been steadily accelerating over the last 40 years. Therefore, non-emergency patients are believed to abuse the emergency service [4]. In terms of patients, however, the lack of other health care units is claimed to be the first reason for the emergency department admissions [5]. The present case study aims to investigate a patient with a large number of admissions to the adult emergency service at the Sakarya University Training and Research Hospital, to understand the cost of the patient to the public through the number of admissions and the invoice amount, and to reveal the solutions available for such patients.

\section{Case Presentation}

A 34-year-old male patient. He has a known history of antisocial personality

*Address for Correspondence: Durmuş E, Department of Medicine, Sakarya Ministry of Health Sakarya University, Turkey, E-mail: muhalefet@hotmail.com

Copyright: (C) 2020 Durmuş E. This is an open-access article distributed under the terms of the Creative Commons Attribution License, which permits unrestricted use, distribution, and reproduction in any medium, provided the original author and source are credited.

Received 12 June 2020; Accepted 19 June 2020; Published 26 June 2020 disorder, substance abuse and alcohol abuse. He is single and lives with his parents. Although he's been working in part-time jobs, he doesn't have a fulltime job. According to his medical history, the patient's domestic relations were bad, he was constantly arguing with his father, and he did not love his father. He said his relationship with other relatives, especially with his uncles were bad. The patient does not have a known history of other diseases or any medication that he uses continuously. He stated that he was on drugs, regardless of the type, that his sex life was bad, that he had not been in a relationship for a long time. When the records from the hospital system between 01/01/2018 and $12 / 31 / 2019$ were examined, it was seen that the patient has admitted to the hospital adult emergency service 126 times in 2018, 140 times in 2019, 266 times in total, hospitalized 4 times, and referred 9 times to intensive care since there were no empty beds in the hospital. His hospitalization days in our hospital were between 1-4 days, and 7 days in total. It was found that the patient was admitted to the emergency room for different reasons, especially for abdominal pain, thinner abuse, excessive drug use, and self-harm. In Sakarya University Training and Research Hospital alone, the total invoice for the examinations and treatments for the patient was 21,763.05 Turkish Lira.

\section{Discussion}

Emergency services are hospital units where all types of cases can be admitted 24/7 and where cases with the life-threatening risks are admitted and treated. However, these units have also been started to be overused by non-emergency patients in recent years. In their study, Renee Gindi et al. investigated adult patients admitted to the emergency service, without hospitalization, in the last 12 , and found that the first three reasons for admission were the fact that it was the only hospital, and doctors' offices were closed, and that there wasn't another place for admission [5]. Goldbeck et al. also report that those with alcohol and drug addiction are more likely to admit to the hospital again [6]. Annie Herbert et al., however, pointed out that it is necessary to develop strategies to reduce such admission in the future, especially after the admissions of near-adult adolescents, admitted because of violence, addiction and self-harm $[7,8]$. Our case was constantly admitting to the emergency service for reasons of self-harm, such as excessive drug use, thinner abuse, and his number of admissions was increasing compared to the 
previous year. The fact that our patient admitted to the emergency service 266 times in two years, and that his examination and treatment costs reached $21,763.05 \mathrm{TL}$, supports the above-mentioned studies, indicating the abuse of emergency services, and increasing costs. In the study of Daniel Weisz et al., patients admitted to the primary care and emergency service were studied, and it was found that $19 \%$ of the patients who had admitted the emergency service did not have a health insurance. The fact that the emergency service is being used continuously by our patient, who is unemployed, and has no social security, confirms the findings of above-mentioned study since the emergency services are free of charge in case of emergency in Turkey. It is seen that these patients with very frequent admissions increase the emergency service workload. Paying more attention for such patients than normal patients, addressing these patients in the outpatient clinic settings in particular, and analyzing and solving the underlying causes can help eliminate the source of the problem.

\section{Conclusion}

Patients with addictions and psychiatric disorders have the potential to harm themselves and cause multiple emergency service admissions, leading to increased workload in the emergency service. Such patients may need detailed psychiatric examination and follow-up. Otherwise, these patients may continue to harm themselves and have a negative impact on the workload of the emergency service.

\section{References}

1. Thomas E. Cowling and Azeem Majeed. "Overuse of Emergency Departments." JAMA 309 (2013): 2549.

2. Seyhan Çil Koçyiğit, Gökçen Şenel and Hüseyin Bostanci. "Expectations of Health Sector from Accounting Education and Practice of Ankara Public Hospitals." J Contemp Med 17 (2016): 547.

3. Lakshini Gunasekera, Hamed Akhlaghi, Christina Sun-Edelstein and John Heywood, et al. "Overuse of Opioids for Acute Migraine in an Australian Emergency Department." Emerg Med 36 (2006): 1328.

4. Adams, James. "Emergency Department Overuse: Perceptions and Solutions." JAMA 309 (2013): 1173.

5. Robin A. Cohen and Michael E. Martinez. "Emergency Room Use Among Adults Aged 18-64: Early Release of Estimates From the National Health Interview Survey." Induc Dis 18 (2008): 11.

6. Inbal Shlomi Polachek, Kinwah Fung and Simone N. Vigod. "Alcohol and Drug Misuse, Risk of Re-admission to a General Hospital and Psychiatric Contact." Scott Med J 57 (2012): 1-5.

7. Annie Herbert, Ruth Gilbert, Arturo González-Izquierdo and Alexandra Pitman et al. "Risks of Death and Emergency Re-admission in Adolescents Hospitalised with Violent, Drug- or Alcohol-Related, or Self-Inflicted Injury: A Population-Based Cohort Study." PLOS Med 12 (2015): 1001-1931.

8. Weisz, Daniel. "Emergency Department Use: A Reflection of Poor Primary Care Access." Am J Manag Care 21 (2015): 152-160.

How to cite this article: Ensar Durmus. "Emergency Department Overuse: A Case Report." Clin Case Rep 10 (2020): 1363. DOI: 10.37421/jccr.2020.10.1363 